\title{
Desempenho agronômico de acessos de mandioca de mesa em área de Cerrado no município de Unaí, região noroeste de Minas Gerais
}

\section{Agronomic performance of sweet cassava germplasm accesses in Cerrados area of Unaí County, Northeast region of Minas Gerais}

\author{
Eduardo Alano VIEIRA ${ }^{1}$; Josefino de Freitas FIALHO²; Luiz Joaquim Castelo Branco CARVALHO ${ }^{3}$; \\ Juaci Vitoria MALAQUIAS ${ }^{4}$; Francisco Duarte FERNANDES ${ }^{2}$ \\ ${ }^{1}$ Autor para correspondência. Pesquisador Doutor; Embrapa Cerrados, BR 020 km 18, CEP 73310-970, Planaltina-DF. \\ eduardo.alano@embrapa.br \\ ${ }^{2}$ Pesquisador Mestre; Embrapa Cerrados; josefino.fialho@embrapa.br; francisco.fernandes@embrapa.br \\ 3 Pesquisador Doutor; Embrapa Recursos Genéticos e Biotecnologia, luiz.castelo@embrapa.br \\ ${ }^{4}$ Analista Bacharel; Embrapa Cerrados; juaci.malaquias@embrapa.br
}

Recebido em: 30-04-2014; Aceito em: 11-05-2015

\section{Resumo}

A região noroeste de Minas Gerais apresenta como característica o grande número de propriedades rurais de agricultura familiar. Nessa região, o cultivo de mandioca de mesa apresenta potencial para expansão em razão da rentabilidade e da proximidade do município ao mercado consumidor do Distrito Federal. Entretanto, o cultivo da mandioca na região ocorre com baixo emprego de tecnologias. Dessa forma, o objetivo do estudo foi determinar o desempenho agronômico de oito acessos-elite de mandioca de mesa no município de Unaí-MG. Os experimentos foram conduzidos entre novembro de 2010 e março de 2012 (safra 2010/2012), e entre novembro de 2011 e março de 2013 (safra 2011/2013), em área experimental da Escola Agrícola de Unaí. O delineamento experimental empregado foi o de blocos completos, com tratamento casualizados, com três repetições. Foram documentadas informações da altura da primeira ramificação, da altura da planta, do peso da parte aérea, da produtividade de raízes tuberosas, da porcentagem de amido e do tempo de cocção das raízes. Os dados foram submetidos à análise de variância individual e conjunta, e ao teste de agrupamento de médias. Os resultados revelaram que: i) os acessos diferiram quanto a todas as características avaliadas; ii) o fator safra influenciou sobre o desempenho dos acessos quanto a todas as características avaliadas, exceto altura da planta; iii) todos os acessos avaliados apresentaram potencial agronômico para cultivo na região; e iv) o acesso BGMC 751 (BRS Japonesa) revelou elevado desempenho agronômico, com destaque para produtividade e tempo de cocção das raízes tuberosas.

Palavras-chave adicionais: aipim; competição de cultivares; macaxeira; Manihot esculenta Crantz; melhoramento genético

\begin{abstract}
The Northeast Region of the state of Minas Gerais, Brazil, presents special characteristics with a large number of family farms. Table sweet cassava presents high potential to be incorporated in this agriculture system for reasons such yield potential and proximity to the large consumer market of Federal District. However, the cultivation of cassava in the region occurs with low use of technologies. Thus, the aim of this study was to determine the agronomic performance of eight elite accessions of sweet cassava in the municipality of Unai (MG). The experiments were conducted between November 2010 and March 2012 (season 2010/2012) and between November 2011 and March 2013 (season 2011/2013) in an experimental field of the Agricultural School of Unaí. The experimental design used was a complete block design with randomized treatments, with three replications. Information of the height of the first branch, plant height, weight of shoot, root yield, percentage of starch in tuberous roots and time for cooking the roots were documented. Data were subjected to analysis of individual and joint variance and mean test group. The results revealed that i) the accessions differed in all variables, ii) crop factor influenced the performance of accesses as all traits except plant height; iii) all accessions showed agronomic potential for cultivation in this region, and iv) the BGMC 751 access (BRS Japanese) revealed high agronomic performance, especially productivity and time for cooking the tuberous roots.
\end{abstract}

Additional keywords: "aipim"; cultivar competition; "macaxeira”; Manihot esculenta Crantz; plant breeding 


\section{Introdução}

A região noroeste de Minas Gerais possui grande número de propriedades rurais de agricultura familiar e de assentamentos de reforma agrária. Especificamente no Município de Unaí, existem 3.593 estabelecimentos rurais, dos quais 2.731 (76,1\%) são de agricultura familiar (IBGE, 2011), onde se destacam combinações de cultivos agrícolas e criações (Oliveira et al., 2009). Nessas condições de cerrado, a cultura da mandioca é utilizada como um dos componentes dos complexos sistemas de produção utilizados pelos produtores, constituindo uma opção relevante na estratégia de segurança alimentar de suas famílias e na diversificação da alimentação animal, contribuindo como uma das fontes de renda familiar. O cultivo de mandioca de mesa apresenta potencial para expansão na região, em razão da rentabilidade da atividade e da proximidade do município de Unaí ao grande mercado consumidor do Distrito Federal.

Entretanto, o cultivo da mandioca de mesa na região ocorre com baixo emprego de tecnologias, e as variedades utilizadas, normalmente, não passaram por um processo formal de melhoramento genético, sendo suscetíveis à bacteriose e com baixo potencial genético de produção de raízes tuberosas. Tal condição resulta em baixa produtividade e na qualidade culinária das raízes tuberosas, o que dificulta o acesso a mercados mais exigentes. Esses mercados exigem raízes tuberosas com baixo tempo de cozimento e boas qualidades culinárias (Borges et al., 2002; Fialho et al., 2009), entre as quais estão a textura das raízes tuberosas, a plasticidade e a pegajosidade da massa cozida, que estão diretamente relacionadas ao tempo de cozimento das raízes (Lorenzi, 1994). Dessa forma, o tempo de cozimento pode ser utilizado como critério indicativo da qualidade culinária da massa cozida nas raízes.

Em experimentos conduzidos no Distrito Federal e no entorno, ao avaliarem 11 variedades de mandioca de mesa, em sete locais, por duas safras, Vieira et al. (2009) relataram elevada variabilidade em relação ao tempo para a cocção e para a produtividade de raízes tuberosas, sendo detectada variação de 11 a 29 minutos e de 22.130 a $53.120 \mathrm{~kg} \mathrm{ha}^{-1}$, respectivamente. Concluíram que os fatores variedades, locais e anos influenciaram no desempenho das variedades, indicando que a expressão dos caracteres de produção e a qualidade culinária das raízes tuberosas, nas variedades de mandioca de mesa, estão fortemente relacionadas ao genótipo e às condições edafoclimáticas do local de cultivo, exigindo a execução de trabalhos de pesquisa de melhoramento e de seleção de variedades nas diferentes regiões de plantio (Fukuda et al., 2002; Ceballos et al., 2004).

Dessa forma, o objetivo do estudo foi determinar o comportamento agronômico de oito acessos-elite de mandioca de mesa em área de
Cerrado, no município de Unaí, região noroeste de Minas Gerais.

\section{Material e métodos}

Os experimentos de campo foram conduzidos entre novembro de 2010 e março de 2012 (safra 2010/2012), e entre novembro de 2011 e março de 2013 (safra 2011/2013), em área experimental da Escola Agrícola de Unaí, localizada no município de Unaí, em Minas Gerais (16은 32,410' S e 46은 50,691' W; $644 \mathrm{~m}$ de altitude). O solo do local foi classificado como Latossolo Vermelho distrófico (Embrapa, 1999). O clima, conforme a classificação de Köppen, é do tipo Aw, ou seja, tropical com estação seca. Foram avaliados oito acessos de mandioca de mesa, com coloração creme da polpa das raízes, mantidos no Banco Regional de Germoplasma de Mandioca do Cerrado (BGMC) (Tabela 1). O delineamento experimental foi o de blocos casualizados, com três repetições. Dentre os acessos avaliados, BGMC 982 (IAPAR 19/Pioneira), BGMC 753 (IAC 576-70/Japonesinha), BGMC 1289 (BRS Moura) e BGMC 751 (BRS Japonesa) são variedades indicadas para o cultivo na região do Distrito Federal (Vieira et al., 2009; Fialho et al., 2009; Vieira et al., 2011), tendo sido os demais acessos selecionados para o estudo, pelo fato de apresentarem potencial produtivo e coloração creme da polpa das raízes. Cada parcela foi composta por 4 linhas com 10 plantas, em espaçamento de $1,20 \mathrm{~m}$ entre as linhas e $0,80 \mathrm{~m}$ entre as plantas, sendo a área útil de cada parcela constituída pelas 16 plantas centrais $\left(15,36 \mathrm{~m}^{2}\right)$. Todos os tratos culturais foram realizados de acordo com as recomendações técnicas da cultura (Fialho et al., 2013; Fialho \& Vieira, 2013).

As colheitas de raízes foram feitas dezesseis meses após o plantio nas duas safras. Nesta época, foram avaliadas, em todas as plantas da área útil, a altura de plantas (AP), em $\mathrm{m}$; a altura da primeira ramificação (APR), em m; o peso da parte aérea sem a cepa (PPA), em $\mathrm{kg} \mathrm{ha}^{-1}$; a produtividade de raízes tuberosas (PR), em $\mathrm{kg} \mathrm{ha}^{-1}$; a porcentagem de amido por meio do método da balança hidrostática (AM) descrito por Grosmann \& Freitas (1950), e o tempo para a cocção (TC), em minutos, de acordo com o método descrito por Borges et al. (2002).

Os dados obtidos foram primeiramente submetidos à análise de variância individual para a determinação da homogeneidade das variâncias dos erros experimentais entre as safras. Atendido 0 pressuposto de homogeneidade de variâncias dos erros experimentais (Gomes, 1991), foi procedida a análise de variância conjunta dos experimentos. As médias das variáveis respostas foram agrupadas por meio do teste Scott \& Knott, com 5\% de probabilidade de erro. As análises estatísticas foram realizadas com o auxílio dos programas estatísticos SAS (Statistical Analysis System - versão 9.1.3) (SAS Institute 2006) e Genes (Cruz, 2001). 
Tabela 1 - Acessos de mandioca de mesa analisados com respectivos códigos do banco regional de Germoplasma de mandioca do Cerrado (BGMC), nomes comuns e locais de coleta/procedência (LCP). Sweet cassava accessions of this study with the respective codes of the regional cassava germoplasm bank of the Cerrado (BGMC), popular names and sampling sites/origin (LCP).

\begin{tabular}{lll}
\hline Acessos & \multicolumn{1}{c}{ Nome comum } & \multicolumn{1}{c}{ LCP } \\
\hline BGMC 982 & lapar 19/Pioneira & IAPAR - Londrina (PR) \\
BGMC 753 & IAC 756-70/Japonesinha & IAC - Campinas (SP) \\
BGMC 1289 & BRS Moura/Taquara-Amarela & Embrapa Cerrados - Brasília (DF) \\
BGMC 751 & BRS Japonesa/Japonesa & Embrapa Cerrados - Brasília (DF) \\
BGMC 1328 & Não possui denominação & Brasília (DF) \\
BGMC 768 & Não possui denominação & Luziânia (GO) \\
BGMC 1243 & Amarela-Rio & Brasília (DF) \\
BGMC 1295 & Vassourinha-Amarela & Brasília (DF) \\
\hline
\end{tabular}

\section{Resultados e discussão}

Como os quocientes obtidos por meio da relação entre o maior e o menor quadrado médio dos resíduos das análises de variância individuais dos caracteres se mostraram inferiores a sete, indicando a existência de homogeneidade nas variâncias dos erros experimentais, procedeu-se à análise conjunta dos dados (Gomes, 1991).

A análise de variância conjunta apontou a existência de diferenças entre as médias das safras de 2010/2012 e 2011/2013 das características APR, PPA e AM (Tabelas 2 e 3), indicando que a média dessas características foi influenciada pelo fator safra. Entretanto, o fator safra não influenciou na média das características AP, PR e TC (Tabelas 2 e 3). Os resultados obtidos no presente trabalho concordam parcialmente com os encontrados por Vidigal Filho et al. (2000), ao avaliarem variedades de mandioca de indústria, no noroeste do Paraná, por três safras, que relataram a influência do fator safra na manifestação das médias das características AP, APR, AM, PPA e PR. Por sua vez, Vieira et al. (2009), ao avaliarem acessos de mandioca de mesa, em seis áreas de Cerrado do Distrito Federal, por duas safras, detectaram a influência do fator safra sobre a característica PR em apenas um dos locais, e que em nenhum dos seis locais em que os experimentos foram conduzidos, o fator safra influenciou no TC.

Dentre as características estudadas, não foram detectadas diferenças entre as médias dos acessos nas duas safras apenas para a característica PR (Tabelas 2 e 3); portanto, todas as demais características avaliadas apresentaram variação no grupo de acessos avaliados. Entretanto, foi detectada a presença de interação entre os fatores safra e acessos para todas as características, com exceção da AP (Tabelas 2 e 3 ).

Tabela 2 - Resumo da análise de variância conjunta, média e coeficiente de variação (CV\%) das características altura da primeira ramificação (APR), altura da planta (AP), peso da parte aérea sem a cepa (PPA), produtividade de raízes (PR), amido nas raízes (AM) e tempo para a cocção (TC), avaliados em oito acessos de mandioca de mesa, nas safras de 2010/2012 (S1) e 2011/2013 (S2), em Unaí-MG. Summary of combined analysis of variance, mean and the coefficient of variation (CV\%) of the traits height of first branching (APR), plant height (AP), root yield (PR), root starch (AM) and cooking time (TC) evaluated in eight sweet cassava accessions, in seasons 2010/2012 (S1) and 2011/2013 (S2) in Unaí-MG.

\begin{tabular}{lcccrrrr}
\hline \multirow{2}{*}{ Fator de variação } & \multirow{2}{*}{${ }^{(1)}$ GL } & \multicolumn{7}{c}{ Quadrado médio } \\
\cline { 3 - 8 } & & APR & AP & PPA & PR & AM & TC \\
\hline Blocos/Safra (B/S) & 4 & 0,003 & $0,090^{*}$ & 9.936 .260 & 18.794 .201 & 0,67 & 3,10 \\
Safras (S) & 1 & $0,035^{*}$ & 0,030 & $80.350 .303^{*}$ & 2.059 & $29,06^{*}$ & 15,68 \\
Acessos (A) & 7 & $0,181^{*}$ & $0,186^{*}$ & $270.509 .323^{*}$ & 19.296 .675 & $9,00^{*}$ & $36,15^{*}$ \\
A x S & 7 & $0,016^{*}$ & 0,011 & $20.941 .225^{*}$ & $46.830 .655^{*}$ & $7,01^{*}$ & $30,10^{*}$ \\
Resíduo (R) & 28 & 0,004 & 0,016 & 5.179 .768 & 9.217 .509 & 0,94 & 4,87 \\
Total & 47 & & & & & & \\
\hline Média & & 0,92 & 2,29 & 29.509 & 37.067 & 28,36 & 22,11 \\
CV (\%) & & 7,20 & 5,60 & 7,71 & 8,19 & 3,42 & 9,98 \\
\hline
\end{tabular}

(1) - Graus de liberdade; * - Significativo a $5 \%$ de probabilidade de erro, pelo teste $\mathrm{F}$. 
Tabela 3 - Níveis de significância (\%), pelo teste F, para o desdobramento da interação acesso x safra, das características: altura da primeira ramificação (APR), peso da parte aérea sem a cepa (PPA), produtividade de raízes (PR), porcentagem de amido nas raízes (AM) e tempo para a cocção (TC), avaliados em oito acessos de mandioca de mesa, nas safras de 2010/2012 e 2011/2013, em Unaí-MG. Significance levels (\%) by $F$ test for the deployment of access $x$ season interaction of the traits height of first branching (APR), shoot weight without original stem cutting (PPA), height of first branching (APR), plant height (AP), root yield (PR), root starch percentage (AM) and cooking time (TC) evaluated in eight sweet cassava accessions, in seasons 2010/2012 (S1) and 2011/2013 (S2) in Unaí-MG.

\begin{tabular}{lcccccc}
\hline Fator & ${ }^{(1)}$ GL & APR & PPA & PR & AM & TC \\
\hline Safras & 1 & 0,87 & 0,05 & 98,82 & 0,10 & 8,37 \\
Acessos & 7 & 0,10 & 0,10 & 7,78 & 0,10 & 0,10 \\
Acessos x safras & 7 & 0,71 & 0,35 & 0,08 & 0,10 & 0,20 \\
Acessos dentro da safra 2010/2012 & 7 & 0,10 & 0,10 & 0,72 & 0,30 & 2,88 \\
Acessos dentro da safra 2011/2013 & 7 & 0,10 & 0,10 & 0,68 & 0,10 & 0,10 \\
Resíduo & 18 & & & & & \\
Total & 47 & & & & & \\
\hline
\end{tabular}

(1) - Graus de liberdade.

Também foram detectadas diferenças entre os acessos para todas as características avaliadas dentro das safras (Tabelas 2 e 3 ), indicando que dentro dos anos houve variação entre os acessos.

Dentre os acessos avaliados, os que apresentaram médias superiores aos demais quanto à característica APR, em ambas as safras, foram BGMC 768 e BGMC 982 (Tabela 4). Para a característica $A P$, os acessos que apresentaram médias significativamente superiores aos demais foram BGMC 768, BGMC 982, BGMC 1328 e BGMC 753 (Tabela 4). Estas características agronômicas são importantes quando da seleção de acessos por se relacionarem diretamente com a facilidade de tratos culturais, disponibilidade de ramas para novos plan- tios, facilidade de plantio mecanizado e facilidade de colheita. Dessa forma, os acessos preferidos são aqueles que apresentam maior altura da primeira ramificação ou que não apresentem ramificação e que apresentem elevada altura de planta (Fukuda et al., 2002; Vieira et al., 2013). Desde que a altura não seja excessiva (superior a $3 \mathrm{~m}$ ), o que pode propiciar acamamento de plantas em áreas com solos férteis e sujeitas a ventos fortes, como relatado por Otsubo et al. (2009) ao avaliarem cultivares de mandioca de indústria em área de Cerrado no Mato Grosso do Sul. Este problema não foi detectado no presente trabalho, onde a maior altura de planta observada foi de $2,52 \mathrm{~m}$ para os acessos BGMC 768 e BGMC 982, na safra de 2012/2012.

Tabela 4 - Comparação de (1)médias das características altura da primeira ramificação (APR), altura da planta (AP) e peso da parte aérea sem a cepa (PPA), avaliados em oito acessos de mandioca de mesa, nas safras de 2010/2012 e 2011/2013, em Unaí-MG. Comparison of means of the traits height of first branching $(A P R)$, plant height $(A P)$ and shoot weight without original stem cutting (PPA) evaluated in eight sweet cassava accessions, in seasons 2010/2012 (S1) and 2011/2013 (S2) in Unaí-MG.

\begin{tabular}{lccccc}
\hline \multirow{2}{*}{ Acessos } & \multicolumn{2}{c}{ APR } & \multicolumn{2}{c}{ AP } & \multicolumn{2}{c}{ PPA } \\
\cline { 2 - 6 } & $2010 / 2012$ & $2011 / 2013$ & $2010 / 2012 \mathrm{e} \mathrm{2011/2013}$ & $2010 / 2012$ & $2011 / 2013$ \\
\cline { 2 - 6 } & $--------(\mathrm{m})$ & $---------\left(\mathrm{kg} \mathrm{h}^{-1}\right)-------$ \\
\hline BGMC 1328 & $0,63 \mathrm{c}$ & $0,76 \mathrm{~b}$ & $2,44 \mathrm{a}$ & $31.399 \mathrm{~b}$ & $30.104 \mathrm{~b}$ \\
BGMC 751 & $0,91 \mathrm{~b}$ & $0,87 \mathrm{~b}$ & $2,22 \mathrm{~b}$ & $33.458 \mathrm{~b}$ & $30.231 \mathrm{~b}$ \\
BGMC 753 & $0,85 \mathrm{~b}$ & $0,78 \mathrm{~b}$ & $2,33 \mathrm{a}$ & $24.264 \mathrm{c}$ & $23924 \mathrm{c}$ \\
BGMC 768 & $1,10 \mathrm{a}$ & $1,29 \mathrm{a}$ & $2,50 \mathrm{a}$ & $47.964 \mathrm{a}$ & $41.174 \mathrm{a}$ \\
BGMC 982 & $1,11 \mathrm{a}$ & $1,22 \mathrm{a}$ & $2,50 \mathrm{a}$ & $28.209 \mathrm{c}$ & $27.396 \mathrm{~b}$ \\
BGMC 1243 & $0,78 \mathrm{~b}$ & $0,92 \mathrm{~b}$ & $2,15 \mathrm{~b}$ & $27.236 \mathrm{c}$ & $27173 \mathrm{~b}$ \\
BGMC 1289 & $0,88 \mathrm{~b}$ & $0,84 \mathrm{~b}$ & $2,06 \mathrm{~b}$ & $25.174 \mathrm{c}$ & $26514 \mathrm{~b}$ \\
BGMC 1295 & $0,86 \mathrm{~b}$ & $0,87 \mathrm{~b}$ & $2,12 \mathrm{~b}$ & $28.716 \mathrm{c}$ & $19.201 \mathrm{~d}$ \\
\hline Média & 0,89 & 0,94 & 2,29 & 30.802 & 28.214 \\
(2)Amplitude & 0,48 & 0,53 & 0,44 & 23.700 & 21973 \\
\hline
\end{tabular}

(1) - Médias seguidas pela mesma letra minúscula na vertical não diferem entre si, a $5 \%$ de probabilidade de erro, pelo teste de agrupamento de médias de Scott e Knott; (2) - Diferença entre a maior e a menor média. 
Quanto à característica PPA, apenas o acesso BGMC 768 teve médias superiores aos demais em ambas as safras (Tabela 4). Esta característica é importante no momento da seleção de acessos por estar relacionada à possibilidade de utilização da parte aérea de mandioca como fonte de proteína na alimentação animal (Souza et al., 2011), bem como para o fornecimento de ramas para o estabelecimento de novos plantios e cobertura do solo (controle de erosão, manutenção de umidade no solo e controle de ervas daninhas) (Fukuda et al., 2002; Vieira et al., 2013).

Para a característica AM na safra de 2010/2011, os acessos que apresentaram médias superiores aos demais foram BGMC 982, BGMC 1243 e BGMC 1289 e na safra de 2012/2013 foram os acessos BGMC 1243, BGMC 982, BGMC 751, BGMC 753, BGMC 1328 e BGMC 768 (Tabela 5). O AM, apesar de ser mais importante na seleção de materiais para a indústria de farinha e fécula, em função de, juntamente com a produtividade de raízes, determinar o potencial produtivo de determinado acesso, tem importância na seleção de acesso de mandioca de mesa quando se pensa no aproveitamento das raízes para duplo propósito (mesa e indústria).

Tabela 5 - Comparação de (1)médias das características produtividade de raízes em (PR), porcentagem de amido nas raízes (AM) e tempo para a cocção (TC), avaliados em oito acessos de mandioca de mesa, nas safras de 2010/2012 e 2011/2013, em Unaí-MG. Comparison of means of the traits root yield (PR), root starch (AM) and cooking time (TC) evaluated in eight sweet cassava accessions, in seasons 2010/2012 (S1) and 2011/2013 (S2) in Unaí-MG.

\begin{tabular}{lcccccc}
\hline \multirow{2}{*}{ Acessos } & \multicolumn{2}{c}{ PR } & \multicolumn{2}{c}{ AM } \\
\cline { 2 - 7 } & 2010/2012 & $2011 / 2013$ & $2010 / 2012$ & $2011 / 2013$ & $2010 / 2012$ & $2011 / 2013$ \\
\cline { 2 - 7 } & $---------\left(\mathrm{kg} \mathrm{ha}^{-1}\right)---------$ & $--------(\%)$ & -------- & ------- & $(\mathrm{min})--------$ \\
\hline BGMC 1328 & $34.696 \mathrm{~b}$ & $40.104 \mathrm{a}$ & $26,48 \mathrm{c}$ & $27,57 \mathrm{a}$ & $24,00 \mathrm{a}$ & $19,57 \mathrm{c}$ \\
BGMC 751 & $43.986 \mathrm{a}$ & $37563 \mathrm{a}$ & $28,84 \mathrm{~b}$ & $28,61 \mathrm{a}$ & $18,00 \mathrm{~b}$ & $17,32 \mathrm{c}$ \\
BGMC 753 & $35.625 \mathrm{~b}$ & $35.667 \mathrm{~b}$ & $28,86 \mathrm{~b}$ & $28,46 \mathrm{a}$ & $22,00 \mathrm{a}$ & $22,48 \mathrm{~b}$ \\
BGMC 768 & $36554 \mathrm{~b}$ & $34.507 \mathrm{~b}$ & $28,42 \mathrm{~b}$ & $27,50 \mathrm{a}$ & $24,00 \mathrm{a}$ & $23,00 \mathrm{~b}$ \\
BGMC 982 & $35854 \mathrm{~b}$ & $39.861 \mathrm{a}$ & $30,94 \mathrm{a}$ & $29,30 \mathrm{a}$ & $20,33 \mathrm{~b}$ & $19,70 \mathrm{c}$ \\
BGMC 1243 & $37.007 \mathrm{~b}$ & $34028 \mathrm{~b}$ & $30,22 \mathrm{a}$ & $29,51 \mathrm{a}$ & $22,33 \mathrm{a}$ & $21,76 \mathrm{~b}$ \\
BGMC 1289 & $33.382 \mathrm{~b}$ & $41.979 \mathrm{a}$ & $30,03 \mathrm{a}$ & $25,54 \mathrm{~b}$ & $19,67 \mathrm{~b}$ & $28,47 \mathrm{a}$ \\
BGMC 1295 & $39.486 \mathrm{a}$ & $32.778 \mathrm{~b}$ & $29,31 \mathrm{~b}$ & $24,17 \mathrm{~b}$ & $22,00 \mathrm{a}$ & $29,17 \mathrm{a}$ \\
\hline Média & 37.074 & 37.061 & 29,13 & 27,58 & 21,54 & 22,68 \\
(2)Amplitude & 4.500 & 1.875 & 0,72 & 0,21 & 6,00 & 11,85 \\
\hline
\end{tabular}

(1) - Médias seguidas pela mesma letra minúscula na vertical não diferem entre si, a $5 \%$ de probabilidade de erro, pelo teste de agrupamento de médias de Scott e Knott; (2) - Diferença entre a maior e a menor média.

O teste de agrupamento de médias revelou que, na safra de 2010/2012, os acessos BGMC 751 e BGMC 1295 apresentaram médias superiores aos demais acessos avaliados para a característica PR, e na safra de 2011/2013, os acessos BGMC 1289, BGMC 1328, BGMC 982 e BGMC 751 foram superiores aos demais (Tabela 5). Para a característica TC, na safra de 2010/2012, os acessos BGMC 751, BGMC 1289 e BGMC 982 apresentaram médias inferiores aos demais, enquanto na safra de 2011/2013 foram os acessos BGMC 751, BGMC 1328 e BGMC 982, que tiveram os menores TCs (Tabela 5). Ambas as características são de fundamental importância para a seleção de acessos de mandioca de mesa, uma vez que determinam o potencial produtivo dos mesmos e a qualidade culinária das raízes tuberosas produzidas no local de condução do experimento (Lorenzi, 1994; Fukuda et al., 2002; Ceballos et al., 2004).

Dessa forma, é possível afirmar que, no grupo de acessos avaliados, todos apresentam potencial para cultivo na região de Unaí (MG), uma vez que: i) todos os acessos revelaram produtividade de raízes tuberosas superiores a $30 \mathrm{t} \mathrm{ha}^{-1}$, em ambas as safras, o que é muito superior à média desta característica na região do Cerrado, que é de $13 \mathrm{t} \mathrm{ha}^{-1}$ (IBGE, 2011); ii) todos os acessos revelaram tempo para 0 cozimento inferiores a 30 minutos, que é o tempolimite aceitável para a comercialização de mandioca de mesa (Lorenzi, 1994; Fukuda et al., 2002); iii) todos os acessos apresentaram arquitetura razoável, uma vez que nenhum ramificou abaixo de $60 \mathrm{~cm}$ ou apresentou estatura inferior a $2 \mathrm{~m}$, nem superior a $3 \mathrm{~m}$ (Fukuda et al., 2002; Otsubo et al., 2009; Vieira et al., 2013); e iv) todos os acessos apresentaram produtividade de parte aérea superiores a $19 \mathrm{t} \mathrm{ha}^{-1}$, o que pode ser considerado boa produtividade de forragem, uma vez que a parte aérea de mandioca apresenta, em média, $13 \%$ de proteína bruta (Souza et al., 2011).

O acesso BGMC 751 (variedade BRS Japonesa, recomendada para o cultivo na região do Distrito 
Federal) (Vieira et al., 2011), merece destaque em função do desempenho agronômico apresentado, pois teve elevado desempenho agronômico nas safras, visto que, dentre as características avaliadas, apenas a produtividade de raízes tuberosas foi significativamente diferente entre as safras (Tabelas 3; 4 e 5). Ademais, esse acesso teve elevadas médias de produtividade de raízes tuberosas $\left(43.986 \mathrm{~kg} \mathrm{ha}^{-1} \mathrm{e}\right.$ $37.563 \mathrm{~kg} \mathrm{ha}^{-1}$ nas safras de 2010/2012 e 2011/2013, respectivamente) e baixos tempos para o cozimento, em ambas as safras. Entretanto, antes da recomendação de qualquer um dos acessos avaliados para o plantio comercial na região Noroeste de Minas Gerais, seria interessante a validação do desempenho dos mesmos em maior número de locais por meio da utilização da metodologia da seleção participativa de variedades de mandioca (Fialho \& Vieira, 2011), na qual seriam obtidos, além de dados agronômicos, informações relativas à preferência dos produtores.

\section{Conclusões}

O fator safra influenciou no desempenho dos acessos quanto a todos os caracteres avaliados, exceto para a altura da planta, evidenciando a necessidade de avaliações dos mesmos em diferentes anos e locais.

Todos os acessos avaliados apresentaram potencial agronômico para cultivo na região de UnaíMG.

O acesso BGMC 751 (BRS Japonesa) tem elevado desempenho agronômico entre as safras, com destaque para a produtividade de raízes tuberosas e tempo para a cocção das raízes.

\section{Agradecimentos}

Os autores agradecem à Empresa Brasileira de Pesquisa Agropecuária (Embrapa), à Fundação Banco do Brasil (FBB) e ao Conselho Nacional de Desenvolvimento Científico e Tecnológico (CNPq), pelo apoio financeiro.

\section{Referências}

Borges MF, Fukuda WMG, Rossetti AG (2002) Avaliação de variedades de mandioca para consumo humano. Pesquisa Agropecuária Brasileira (37)11: 1559-1565. doi: 10.1590/S0100-204X2002001100006.

Ceballos $H$, Iglesias CA, Pérez JC, Dixon AGO (2004) Cassava breeding: opportunities and challenges. Plant Molecular Biology 56(4): 503-516. doi: 10.1007/s11103-004-5010-5.

Cruz CD (2001) Programa genes: aplicativo computacional em genética e estatística. UFV. 648p.

Embrapa - Empresa Brasileira de Pesquisa Agropecuária (1999) Sistema Brasileiro de classificação de solos. Rio de Janeiro/Embrapa Solos. 412p.
Fialho JF, Sousa DMG, Vieira EA (2013) Manejo do solo no cultivo de mandioca. In: Fialho JF, Vieira EA (ed). Mandioca no Cerrado: orientações técnicas, 2 ed. Embrapa Cerrados. p.39-60.

Fialho JF, Vieira EA (2013) Manejo e tratos culturais da mandioca. In: Fialho JF, Vieira EA (ed). Mandioca no Cerrado: orientações técnicas. 2 ed. Embrapa Cerrados. p.61-88.

Fialho JF, Vieira EA (2011) Seleção participativa de variedades de mandioca na agricultura familiar. Embrapa Cerrados. 76p.

Fialho JF, Vieira EA, Silva MS, Moraes SVP, Fukuda WMG, Filho MOSS, Silva KN (2009) Desempenho de variedades de mandioca de mesa no Distrito Federal. Revista Brasileira de Agrociência 15(1-4): 31-35.

Fukuda WMG, Silva SO, Iglesias C (2002) Cassava breeding. Crop Breeding and Applied Biotechnology 2(4): 617-638.

Gomes, FP (1991) Análise de grupos de experimentos. In: Gomes FP (ed). Curso de estatística experimental, 14 ed. Nobel. p.168-197.

Grosmann J, Freitas AG (1950) Determinação do teor de matéria seca pelo método peso específico em raízes de mandioca. Revista Agronômica 14(160-162): 75-80.

IBGE - Instituto Brasileiro de Geografia e Estatística (2011) Sistema IBGE de recuperação automática SIDRA. Disponível em: <http://www.sidra.ibge.gov.br>; Acesso em: 15 out. 2013.

Lorenzi JO (1994) Variação na qualidade culinária das raízes de mandioca. Bragantia 53(2): 237-245.

Oliveira MN, Xavier JHV, Silva FAM, Scopel E, Zoby JLF (2009) Efeitos da introdução do sistema de plantio direto de milho por agricultores familiares do município de Unaí, MG (Cerrado Brasileiro). Pesquisa Agropecuária Tropical 39(1): 51-60.

Otsubo AA, Brito OM, Mercante FM, Otsubo VHN, Gonçalves MA, Telles TS (2009) Desempenho de cultivares elites de mandioca industrial em áreas de cerrado do Mato Grosso do Sul. Semina: Ciências Agrárias 30(4 suplemento 1): 1155-1162. doi: http://dx.doi.org/10.5433/1679-0359.2009v30n4Sup1p1155.

SAS Institute. Statistical analysis system Version 9.1 (2006) SAS Institute. 235p.

Souza AS, Rocha Júnior VR, Mota ADS, Palma MNN, Franco MO, Dutra ES, Santos CCR, Aguiar ACR, Oliveira CR (2011) Valor nutricional de frações da parte aérea de quatro variedades de mandioca. Revista Brasileira de Saúde e Produção Animal 12(2): 441-455. 
Vidigal Filho OS, Pequeno MG, Scapim CA, Vidigal MCG, Maia RR, Sagrilo E, Simon GA, Lima RS (2000) Avaliação de cultivares de mandioca na região noroeste do Paraná. Bragantia 59(1): 69-75. doi: http://dx.doi.org/10.1590/S0006-87052000000100011.

Vieira EA, Fialho JF, Silva MS (2013) Recursos genéticos e melhoramento de mandioca. In: Fialho JF, Vieira EA (ed) Mandioca no Cerrado: orientações técnicas, 2 ed., Embrapa Cerrados. p.27-37.
Vieira EA, Fialho JF, Silva MS, Fukuda WMG, Santos Filho, MOS (2009) Comportamento de genótipos de mandioca de mesa no Distrito Federal. Revista Ciência Agronômica 40(1): 113-122.

Vieira EA, Fialho JF, Silva MS, Paula-Moraes SV, Oliveira CM, Anjos JRN, Rinaldi MM, Fernandes FD, Guimarães Júnior, R (2011) BRS Japonesa: new sweet cassava cultivar for the Distrito Federal region. Crop Breeding and Applied Biotechnology 11(2): 193-196. doi: http://dx.doi.org/10.1590/S1984-70332011000200014. 